\title{
Mythos
}

MYTHOS

Rivista di Storia delle Religioni

$15 \mid 2021$

Varia

\section{Cicerone e le statue degli dèi}

cicero and the Statues of Gods

\section{Claudia Beltrão}

\section{(2) OpenEdition}

Journals

Edizione digitale

URL: https://journals.openedition.org/mythos/3098

DOI: $10.4000 /$ mythos.3098

ISSN: 2037-7746

\section{Editore}

Salvatore Sciascia Editore

\section{Notizia bibliografica digitale}

Claudia Beltrão, «Cicerone e le statue degli dèi», Mythos [Online], 15 | 2021, online dal 15 décembre 2021, consultato il 15 décembre 2021. URL: http://journals.openedition.org/mythos/3098 ; DOI: https://doi.org/10.4000/mythos.3098

Questo documento è stato generato automaticamente il 15 décembre 2021.

Mythos 


\title{
Cicerone e le statue degli dèi
}

\author{
Cicero and the Statues of Gods
}

\author{
Claudia Beltrão
}

1 In anni recenti è maturata una rinnovata attenzione verso il potere religioso e sociale delle immagini degli dèi, che porta a considerarle come testimonianze di varie modalità percettive e come una forma di pensiero e di azione. Le statue, in quanto artefatti prodotti dagli esseri umani, contribuiscono a conferire forme materiali alle divinità. Attribuiscono materialità agli dèi fra gli esseri umani, alimentando la comunicazione, l'emozione, e l'immaginazione in ambito religioso ${ }^{1}$. La Roma di Cicerone era un luogo pieno di dèi, di storie sugli dèi e di immagini che li riguardavano. Ovunque si posassero gli occhi vi erano statue, altari, santuari, e la letteratura romana preserva una vivida tradizione intorno alle controversie sulle immagini divine - controversie che, in molti casi, servirono da modello e da ispirazione per dibattiti e conflitti successivi. La vasta opera di Cicerone è un punto di riferimento imprescindibile per lo studio della storia politica, religiosa e intellettuale di Roma antica: contribuisce a definirne gli spazi, le personalità, le pratiche e le istituzioni, conferendovi forti significati concettuali ${ }^{2}$. Le divinità e le loro immagini sono temi costanti nella sua opera filosofica, nei suoi discorsi e nella sua corrispondenza.

2 La rappresentazione antropomorfica degli dèi è un tema classico, dai dibattiti sugli dèi omerici sino ai canoni della scultura classica ${ }^{3}$. La riflessione su questo problema ha visto un forte rinnovamento negli ultimi decenni, soprattutto sul tema dei corpi degli dèi e la loro percezione e, in particolare, sul luogo specifico della visione e sui riferimenti al concetto e alla percezione degli dèi ${ }^{4}$. In pratica, gli atti della rappresentazione o del riconoscimento di un'immagine divina comportano alcune decisioni di fondo su che cosa la divinità sia e su come essa si manifesti. Nel Mediterraneo antico gli dèi erano in generale concepiti sotto una forma umana, e questo aspetto non è cambiato di molto nel cristianesimo moderno, in tutte le sue diverse versioni ${ }^{5}$. Le divinità erano rappresentate in immagini e il mondo degli dèi era un mondo fisico, materiale. Dèi e dee erano pensati come corpi - esseri immortali in corpi umani o, se non antropomorfici, come corpi scolpiti o, perlomeno, materializzati in un supporto di qualche tipo - non solo rappresentando le divinità, ma conferendo 
loro una presenza significativa ${ }^{6}$. Le logiche religiose e letterarie antiche sfidano semplicemente i comodi rapporti stabiliti dalla razionalità moderna fra l'immagine e il suo referente ${ }^{7}$. Gli dèi sono confinati alla materialità $e$, nel tempo stesso, sono incommensurabili, e i termini di questo dibattito teologico persistono oltre i limiti delle partizioni cronologiche, delle religioni, della geografia e dei contesti culturali.

Nella nostra epoca, caratterizzata dalla multimedialità, le immagini si rivelano un fertile campo di indagine, ma il termine 'idolatria' è investito di una carica negativa ${ }^{8}$. L'idea di 'rappresentazione' ha inoltre un ruolo centrale nel dibattito moderno'. Se la controversia fra Cotta e Velleio nel De Natura Deorum si concentra intorno a ciò che è possibile dire a proposito della divinità a partire dalle tracce della sua immagine, nel contesto romano il concetto di rappresentazione ha limiti precisi ${ }^{10}$; ancora meno efficace è l'idea della 'presenza occasionale' della divinità in una sua immagine, di cui Richard Gordon ha dimostrato la rilevanza per la religione greca, e che Deborah Steiner ha sostenuto parlando di container, vessel, residence, e concetti affini ${ }^{11}$. Questo orizzonte di discorso presuppone una differenza metafisica fra la divinità e l'immagine, ma è anche piuttosto semplificatorio parlare di una presenza pura e semplice. Al contempo, persiste tuttora un problema metodologico nello studio della religione romana: le statue rappresentavano davvero gli dèi? $\mathrm{E}$ in che termini? Il concetto di rappresentazione funziona perfettamente, se si dà la priorità a un'esistenza metafisica degli dèi. Una statua si tramuta nella rappresentazione di qualcosa che va oltre ad essa, indica ciò che non è visibile o non è presente; a rigore, rafforza l'idea secondo cui questo quid esisterebbe necessariamente al di fuori da ciò che lo rappresenta.

Nell'opera di Cicerone, emergono tre modalità principali di rappresentazione in rapporto alle immagini divine, corrispondenti a diversi modi di accostarsi alle statue antropomorfe. Il primo è la statua in quanto oggettivizzazione degli dèi. Di fatto, nell'antichità classica la statua è il principale paradigma della divinità: formidabile strumento di azione rituale e di rappresentazione della divinità in diversi contesti, la statua materializza la presenza divina in modi non necessariamente stabili nel tempo e nello spazio ${ }^{12}$. Senza volerci addentrare nel complesso dibattito sulle 'statue di culto' o sulle 'statue offerte alla divinità', o su 'come identificare le statue di culto nell'antichità', il contesto e, talvolta, il culto sono fondamentali a questo proposito ${ }^{13}$. La seconda modalità è legata a un altro ordine di questioni: se, quando e come la statua si trasformi in un essere animato e attivo ${ }^{14}$; se la statua è vista come una convenzione umana ed è, in certa misura, limitata teologicamente, essa si unisce anche al divino. Una statua si impone in quanto presenza fisica della divinità, ma tale presenza è anche costruita dalla performance dell'oratore. In altre parole, la statua divina è percepita attraverso diversi registri sensoriali e intellettuali. La terza modalità si concentra sulla questione di come gli dèi immortali possano essere conosciuti a partire da un oggetto materiale. Vediamo qui il dibattito teologico sulla tensione tra figura materiale e pensiero sul divino: una discussione di lunghissima durata e di perdurante attualità.

5 In questo lavoro rifletteremo tali modalità ciceroniane di concepire, vedere e riferirsi alle statue divine attraverso tre esempi originali di diversi registri letterari, tratti da altrettanti momenti dell'opera dell'Arpinate. Si tratta di casi di studio complementari, che pertengono alla materialità degli dèi e rivelano varie sfumature delle modalità attraverso cui ci si può riferire alle rappresentazioni antropomorfiche del divino: il primo è quello relativo a una statua di Hermathena acquistata per la villa che Cicerone possedeva a Tuscolo, di cui si fa menzione nella sua corrispondenza; il secondo riguarda 
una statua che si trova al centro di una durissima controversia politica, quella della Libertas di Clodio, di cui si parla nella De Domo; il terzo, analizza un breve passo della controversia sulla forma del divino fra l'accademico Cotta e lo stoico Balbo nel De Natura Deorum. Questi casi di studio rivelano una stretta connessione tra religione, vita politica e dibattito intellettuale nella Roma tardorepubblicana, ponendo specifici problemi relativi alla costruzione, all'uso e alla ricezione delle statue divine.

\section{Hermathena: la materializzazione di Minerva in una nuova Academia}

6 La villa di Cicerone a Tuscolo fa da sfondo a vari dialoghi filosofici di Cicerone nella sua maturità. L'oratore acquistò la villa di Tuscolo e ne seguì il restauro fra il 68 e il 65 a.C., e molte sue lettere permettono di farsi un'opinione informata sulle sue necessità e le sue preferenze $\mathrm{e}^{15}$. Egli costruì due piani, con un ginnasio, detto Accademia, al piano inferiore, dedicato all'attività intellettuale, e una biblioteca in quello più alto, il Liceo, con ovvio richiamo all'insegnamento filosofico di Platone e di Aristotele ${ }^{16}$.

7 Si è molto discusso sull'importanza storica della casa romana in quanto elemento di distinzione sociale e identitario dell'élite romana, e le osservazioni di Cicerone sulle sue residenze sono fonti di grande valore per lo studio di questo tema ${ }^{17}$. Le richieste che egli formulò ad Attico e le statue che egli accolse o rifiutò nella sua collezione sono aspetti largamente studiati da due punti di vista: la strategia retorico-politica volta alla maggior gloria del proprietario, e gli sviluppi del mercato delle opere d'arte nel primo secolo a.C. Si tratta sempre, d'altronde, di immagini divine o di ornamenta legati, in un modo o in un altro, a pratiche o a credenze religiose ${ }^{18}$. Queste immagini si radicano in visioni del modo e sistemi di pensiero: la loro esistenza e la posizione che è loro conferita in uno spazio determinato possono rivelarcene i significati.

Due lettere ad Attico menzionano esplicitamente una di queste opere d'arte, un'erma di Atena, qui detta Minerva ${ }^{19}$ :

Hermathena tua valde me delectat et posita ita belle est ut totum gymnasium eius $\dot{\alpha} v \alpha \dot{\theta} \eta \mu \alpha$ esse videatur. (Att. 1.1.5)

La tua Ermatena è per me fonte di squisito piacere ed è stata collocata in una posizione scelta con tanto buon gusto, che l'intero ginnasio pare ridursi a un

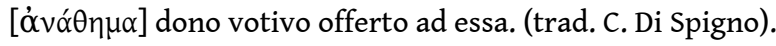

quod ad me de Hermathena scribis, per mihi gratum est. est ornamentum Academiae proprium meae, quod et Hermes commune omnium et Minerva singulare est insigne eius gymnasi. qua re velim, ut scribis, ceteris quoque rebus quam plurimis eum locum ornes. quae mihi antea signa misisti, ea nondum vidi; in Formiano sunt, quo ego nunc proficisci cogitabam. illa omnia in Tusculanum deportabo... (Att. 1.4.3)

Ciò che mi scrivi riguardo all'Ermatena suscita in me enorme piacere. È l'oggetto artistico che va a puntino per la mia Academia, per il fatto che, se generalmente si ricorre alle erme per abbellire i ginnasi, l'effigie di Minerva dà lustro tutto particolare al mio ginnasio. E a tal proposito vorrei che tu, come scrivi, rendessi delizioso quel posticino con il maggior numero possibile di pezzi pregiati. Le statue che mi hai mandato precedentemente, non ho ancora potuto vederle; si trovano nella mia villa di Formia, che ora conto di raggiungere. Le farò trasferire tutte nella 
villa di Tuscolo. (trad. C. Di Spigno, modificata) un oggetto consacrato in un santuario: un'offerta a una divinità ${ }^{20}$. Egli sembra affermare l'intenzione di dedicare tutta la sua Academia alla dea. Nella seconda lettera sostiene che, se in tutti i ginnasi vi è un Hermes, di Minerva si dice che sia «particolarmente appropriata» per lo spazio della sua villa detto Academia. La Hermathena sarebbe dunque un oggetto religioso? Cicerone la definisce un ornamentum Academiae proprium meae. La statua è un óvá́ $\theta \eta \mu \alpha$ e un ornamentum in una nuova Academia. Si tratta di una contraddizione rispetto alla statua di una dea?

10 I tentativi di distinguere fra oggetti rituali e non rituali rivelano la difficoltà insita nello stabilire distinzioni nette, e confermano che la loro funzione dipende dal punto di osservazione, dal campo di applicazione, dalla densità degli elementi simbolici, dalla conoscenza e dalle pratiche religiose inquadrate nel contesto, poiché gli oggetti, come le statue divine possono essere ritualizzati in qualsiasi momento e assumere un significato religioso ${ }^{21}$. La ritualizzazione degli oggetti - specialmente le statue degli dèi - è un Leitmotiv della retorica di Cicerone, che trasforma una statua in un oggetto rituale senza trascurarne il valore estetico e simbolico ${ }^{22}$. Una lettera indirizzata a Fabio Gallo, che acquistò anch'egli delle opere per la villa di Tuscolo, fornisce altre piste sul significato e la funzione della Hermathena per Cicerone:

(...) prorsus enim ex istis emptionibus nullam desidero. tu autem ignarus instituti mei, quanti ego genus omnino signorum omnium non aestimo, tanti ista quattuor aut quinque sumpsisti. Bacchas istas cum Musis Metelli comparas. quid simile? primum ipsas ego Musas numquam tanti putassem atque id fecissem Musis omnibus approbantibus, sed tamen erat aptum bybliothecae studiisque nostris congruens; Bacchis vero ubi est apud me locus? at pulchellae sunt. novi optime et saepe vidi. nominatim tibi signa mihi nota mandassem, si probassem. ea enim signa ego emere soleo quae ad similitudinem gymnasiorum exornent mihi in palaestra locum. Martis vero signum quo mihi pacis auctori? gaudeo nullum Saturni signum fuisse; haec enim duo signa putarem mihi aes alienum attulisse. Mercuri mallem aliquod fuisset.... (Fam. 7.23.2)

Non ho bisogno di alcuno degli acquisti che hai fatto. Poiché non conosci la mia consuetudine, hai acquistato questi quattro o cinque pezzi a un prezzo che considero eccessivo per tutte le statue che hai comprato. Paragoni queste Baccanti alle Muse di Metello. Dov'è la somiglianza? Anzitutto, non ho mai ritenuto che le Muse valessero una somma del genere - e tutte avrebbero approvato questo mio giudizio! Eppure sarebbe stato un buon acquisto per una biblioteca, e uno adeguato ai miei interessi. Ma dove posso mettere le Baccanti? Sono belline, potresti dirmi. Le conosco bene, le ho viste spesso. Avrei dovuto darti un incarico specifico riguardo a statue che conosco, se le avessi gradite. Ho l'abitudine di comprare opere che posso usare per ornare un luogo nella mia palestra, a imitazione dei ginnasi. Ma una statua di Marte! Che cosa posso farne io, che sono un sostenitore di Marte? Sono lieto che non ve ne fosse una di Saturno - avrei pensato che quei due mi avrebbero indebitato! Piuttosto ne preferirei una di Mercurio... (trad. C. Di Spigno)

Le Baccanti, al di là della loro bellezza, non erano appropriate, né Marte poteva essere adatto a chi si definiva pacis auctor. Anche Saturno e Mercurio non avrebbero avuto posto nella Academia, che privilegiava la sapienza e la conversazione filosofica. Nelle Tusculanae, Cicerone dice che era sua abitudine tenere lunghe conversazioni filosofiche nel Liceo, di mattina, e nell'Accademia, al pomeriggio ${ }^{23}$. Si tratta dunque di un contesto 
intellettuale tout court, in cui la composizione e l'arredo dello spazio giocano un ruolo fondamentale. La Hermathena, peraltro, era perfetta in quell'ambito, e tutta l'Accademia le sarebbe stata dedicata, dando forma materiale alla presenza della dea e stimolando la pratica della filosofia. In questi passi Cicerone combina elementi religiosi, estetici e intellettuali, e possiamo vedere come l'immagine religiosa si presti a diversi usi e manipolazioni, alle quali è suscettibile, sia nel contesto rituale che al di fuori di esso.

\section{La pulchra Libertas di Clodio}

Il secondo esempio che si propone qui proviene da un discorso di Cicerone datato all'epoca immediatamente successiva all'esilio, e legato ai suoi conflitti con P. Clodio Pulcro $^{24}$. La De Domo fu pronunciata di fronte al collegio pontificale; il Senato si richiamò a una legge che consentiva il ritorno di Cicerone a Roma, e garantiva la ripresa del possesso delle proprietà che gli erano state confiscate ${ }^{25}$. Si ritenne però opportuno consultare i pontefici riguardo al valore della dedica, fatta da Clodio, da parte della domus nel Palatino alla dea Libertas - secondo Cicerone, per assicurarsi che la proprietà non potesse essere contestata in futuro per ragioni religiose ${ }^{26}$. I pontefici decisero tenere un'udienza delle parti interessate, come in un tribunale. Si trattava di una disputa fra due individui attivi dal punto di vista politico e religioso, che esprimevano posizioni divergenti riguardo alle relazioni con il divino ${ }^{27}$. Discorsi come questo sono media tramite i quali le decisioni potevano essere mutate e le azioni potevano essere guidate; d'altronde, separare il contenuto del discorso dalla sua espressione materiale equivale a perdere di vista il senso dei complessi processi di riflessione dell'oratore e delle tensioni fra l'oratore, il suo pubblico e il contesto generale.

Nel 57, peraltro, Cicerone difese il ripristino della sua casa sul Palatino, la cui area era stata confiscata, a quanto sembra senza fondamento giuridico, e dedicata a Libertas ${ }^{28}$. A quell'epoca, un magistrato e un membro del collegio dei quindecemviri s.f., quale era Clodio, aveva il potere di consacrare un terreno agli dèi, dedicare una statua, e dunque rendere quello spazio sacer ${ }^{29}$. Di fronte ai pontefici, Clodio sottolineò il carattere inviolabile della dedica: un argomento di per sé molto potente. Cicerone, da parte sua, presentò l'azione di Clodio contro la sua proprietà come un affronto alla sua dignitas e alle sue divinità domestiche e, in un'ultima analisi, come un'aggressione a Roma stessa $^{30}$.

14 La strategia principale di Cicerone consiste nel dimostrare che l'azione di Clodio non aveva alcun fondamento religioso, perché violava la corretta prassi romana. D'altra parte, il dedicante era a sua volta indegno di intraprendere un'azione religiosa propriamente intesa ${ }^{31}$. Sino a un certo punto, Cicerone difende con forza un nuovo concetto di religio, nel quale le considerazioni sulla giustizia si sostituiscono a una corretta esecuzione dei rituali ${ }^{32}$. In questo discorso si mescolano questioni di natura politica, religiosa e giuridica di stretta attualità; l'oratore ricorre ad argomenti solidi e, nel contempo, fa uso dell'ironia e del sarcasmo. L'evocazione dello scrupolo religioso e la satira mordace, sono sottolineati dal ricorso al gioco di parole, alla metafora, all'allitterazione. Per tornare alla statua: Clodio consacrò il terreno, eresse un monumento e vi dedicò una statua (consecrasse...monumentum fecisse ... signum dedicasse $)^{33}$. Dal punto di vista lessicale, siamo di fronte a una terminologia rigorosamente religiosa. 
15 La descrizione del progetto di Clodio ha sollevato molti dubbi tra gli studiosi sull'organizzazione e sulle caratteristiche strutturali dello spazio sacro da lui dedicato: si trattava di un altare aperto o un recinto coperto da un tetto. Certo, la presenza della statua indicava che la costruzione era una aedes, cioè la casa di una divinità ${ }^{34}$. È vero che la dedica di un Romano non dipendeva necessariamente dalle statue, ma esse erano oggetti onnipresenti e significativi. Il discorso di Cicerone mette la statua di Libertas al centro dell'argomentazione contro l'iniziativa religiosa di Clodio ${ }^{35}$. La statua sembra essere il signum della consacrazione di cui il terreno di Cicerone era stato oggetto ed egli - come María Emilia Cairo ha recentemente dimostrato - si adopera dunque per squalificarne l'identità in quanto statua di Libertas ${ }^{36}$.

Infatti, le modalità con cui Cicerone si riferisce alla statua sono significative, come si può leggere nel passo qui di seguito riportato:

(...) ista tua pulchra libertas deos penatis et familiaris meos lares expulit, ut se ipsa tamquam in captivis sedibus conlocaret? quid est sanctius, quid omni religione munitius quam domus unius cuiusque civium? hic arae sunt, hic foci, hic di penates, hic sacra, religiones, caerimoniae continentur; hoc perfugium est ita sanctum omnibus ut inde abripi neminem fas sit (Dom. 108).

Codesta tua bella Libertà ha cacciato via i miei dèi Penati e i Lari domestici per prendere posto ella stessa come in un luogo di conquista? Cosa c'è di più sacro, cosa di più protetto da ogni religione della casa di ciascun cittadino? Qui hanno il loro centro gli altari, qui i focolari, qui gli dèi penati, qui i sacrifici, le pratiche religiose, le cerimonie: questo è un asilo sì sacro per tutti, che da esso non è lecito strappare nessuno. (trad. G. Bellardi, con lievi modifiche)

17 La statua agisce ed espelle Cicerone e le sue divinità domestiche dalla sua stessa casa. «Ma che dea è quella che Clodio sostiene sia Libertas?», si domanda quindi l'oratore ${ }^{37}$, che prosegue, nel suo discorso, senza operare una distinzione precisa fra la divinità, la l'idea politica di libertas e la statua di cui racconta la vicenda ricordando la provenienza dalla tomba di una prostituta di Tanagra e il modo in cui venne successivamente fatta oggetto di una serie di appropriazioni illegittime, prima di giungere a Roma ${ }^{38}$.

18 Cicerone denuncia il paradosso per cui il nemico della libertas del cittadino romano si interessa alla Libertas. La Libertas del popolo romano non viene in alcun modo contestata, ma viene messa in dubbio l'identità della statua consacrata nella sua proprietà: si tratta della statua funeraria di una prostituta straniera, che viene accostata a Licentia; allo stesso modo, il suo sedicente tempio sarebbe solo un pretesto che consente a Clodio di erigere una lussuosa costruzione di carattere privato. Da qui la scelta di riferirsi alla statua come ista tua pulchra Libertas, come se la Libertas di Clodio fosse una dea personale. $\mathrm{E}$ a questa statua l'oratore conferisce una capacità di agire: la Libertas di Clodio agisce in quanto statua: Libertatis simulacrum in ea domo... Libertas domo sua.... ${ }^{39}$, espellendo non soltanto Cicerone dalla sua casa, ma anche altre statue divine: $\mathrm{i}$ suoi Lares, i suoi Penates.

19 Vi è ragione di credere che le statue poste in contesti domestici non avessero uno statuto sacro riconosciuto: la tesi di Cicerone in questo passo sembra fondarsi su questo presupposto ${ }^{40}$. L'oratore dovette necessariamente impegnarsi perché la propria argomentazione avesse efficacia persuasiva e presa sul pubblico. Se i Lari e i Penati non erano, a rigore, sacri per i pontefici, per Cicerone essi erano dotati di una sacralità di 
tipo morale. Lo stesso non valeva evidentemente per la Libertas di Clodio. La pulchra Libertas non è la Libertas del popolo romano, non appartiene alla comunità degli dèi romani come i Lares e i Penates di Cicerone, ma ha una forte valenza negativa nella sua argomentazione. Come puntualizza l'oratore: in scelere religio non valet ${ }^{41}$. pone l'accento sul nesso tra religione e diritto, città e impero. Contro le divinità personali egli si pronuncerà duramente anche nel De Legibus: «Il culto degli dèi particolari, siano essi nuovi o stranieri, provoca confusione nei culti e introduce rituali sconosciuti» (suosque deos aut novos aut alienigenas coli confusionem habet religionum, et ignotas caerimonias) ${ }^{42}$. Nella De Domo, peraltro, vediamo una statua animata dall'oratore in maniera simile a una statua di Giove nella Terza Catilinaria, la cui visione nell'area Capitolina guida il console e svela la congiura che minacciava Roma ${ }^{43}$. In questa modalità di confronto con le statue divine, l'idea stessa di divinità e gli aspetti materiali dell'esperienza religiosa coincidono. La perfetta coincidenza fra divinità e statua, peraltro, non è una costante assoluta nell'opera di Cicerone. Ad esempio, nel De Divinatione, Giove lancia un fulmine contro il suo stesso tempio e ruba immagini divine, compresa la sua stessa statua. È questo però un contesto filosofico, che verrà discusso nel nostro terzo e ultimo esempio ${ }^{44}$.

\section{La consuetudo oculorum: immagini e conoscenza degli dèi}

21 Questo terzo caso di studio si lega alla controversia teologica fra i personaggi di Cotta e di Balbo nel De Natura Deorum. In questo dialogo la parola natura indica il proposito di comprendere la divinità in termini fisici ${ }^{45}$. Il famoso passo del primo libro, dove l'accademico Cotta dichiara che gli dèi sono concepiti attraverso l'aspetto che i pittori e gli scultori sceglieranno per immaginarli, rappresenta un problema epistemologico per l'epicureismo: le immagini divine sono contingenti e non forniscono indizi certi per dare fondamento alla rappresentazione antropomorfica degli dèi, sostenuta dall'epicureo Velleio come se fosse un dato universale ${ }^{46}$. Per Cotta, gli esseri umani vivono l'esistenza degli dèi attraverso le loro esperienze con le immagini, le cui forme variano fra i diversi popoli. Se l'antropomorfismo divino è un tema dirimente nel dibattito fra Cotta e Velleio nel primo libro, l'immagine divina non è certo assente dalla discussione della teologia stoica nei libri 2 e 3.

In totale dissenso rispetto alla teologia epicurea, la divinità razionale e provvidenziale degli Stoici non ha forma umana. Nel discorso di Balbo, il dio supremo, legato al principio eterno della «materia», ha creato l'universo per dare alla materia le sue proprietà ${ }^{47}$. Simmetrico, durevole e bello, l'universo fu creato a beneficio degli dèi e degli uomini ${ }^{48}$. Anzi, l'universo e i corpi celesti, di una bellezza perfetta, in una razionale cooperazione con il dio supremo, sono esseri divini, a loro volta ${ }^{49}$. La bellezza perfetta, peraltro, appartiene a figure geometriche come la sfera e il cerchio, ma non a quella umana. Balbo ammette, peraltro, che le immagini degli dèi possano aiutare il vulgus ad approssimarsi a qualche comprensione della natura divina, sebbene in misura imperfetta. D'altronde, così come gli epicurei, anche gli Stoici sostenevano che la nozione «naturale» degli dèi fosse stata contaminata da errori e aspetti irrazionali, promossi dagli stessi poeti e dagli artisti che promuovevano idee corrette intorno agli dèi ${ }^{50}$. Attraverso la consuetudo oculorum creata dalle arti visive, gli esseri umani 
distorsero la verità degli dèi, come dice Balbo:

uidetisne igitur ut a physicis rebus bene atque utiliter inventis tracta ratio sit ad commenticios et fictos deos. quae res genuit falsas opiniones erroresque turbulentos et superstitiones paene aniles. et formae enim nobis deorum et aetates et vestitus ornatusque noti sunt, genera praeterea coniugia cognationes, omniaque traducta ad similitudinem inbecillitatis humanae. [...] haec et dicuntur et creduntur stultissime et plena sunt futilitatis summaeque levitatis (ND 2.70)

Potete ora constatare come partendo da eccellenti ed utili scoperte relative al mondo della natura si sia giunti ad ammettere, come ovvia conclusione, dèi falsi ed immaginari: di qui false opinioni, errori conturbanti e superstizioni poco meno che senili. Abbiamo così imparato a conoscere l'aspetto degli dèi, la loro età, i loro abiti e ì loro ornamenti nonché il loro sesso, i loro matrimoni e i loro rapporti di parentela e il tutto abbassato al livello delle umane debolezze [...] Trattasi di credenze più che sciocche, che rivelano solo un'estrema superficialità e leggerezza. (trad. U. Pizzani)

Il discorso di Balbo si colloca fra teologia, cosmologia ed epistemologia e può essere utilmente messo in relazione con altri passi dell'opera ciceroniana come Lucullus $19 \mathrm{e}$ 30-32, in cui lo stesso Lucullo, con la sua difesa della Vecchia Accademia di Antioco di Ascalona, sottolinea l'inadeguatezza della conoscenza sensibile del mondo visibile ${ }^{51}$.

Per Balbo, dunque, miti e immagini figurative non possono offrire un fondamento sicuro alla conoscenza degli dèi, ma, una volta liberati dagli errori attraverso un'interpretazione stoica dei nomi e dei miti divini, distinguendo gli elementi benefici e malefici delle immagini, possono dare alla collettività dei mortali un qualche sostegno alla conoscenza divina, superando le superstitiones ${ }^{52}$. Le diverse divinità dei vari popoli non sarebbero che nomi e allegorie dell'ordine animato dell'universo, così come le storie tradizionali sugli dèi sarebbero un mezzo attraverso il quale si può conoscere l'ordine dell'universo ${ }^{53}$. In base a questa dottrina, quale sarebbe allora il rapporto fra gli dèi tradizionali di Roma e le loro immagini? Cotta risponde a Balbo, riprendendo il tema della consuetudo oculorum:

itaque quoniam quattuor in partes totam quaestionem divisisti de primaque diximus, consideremus secundam; quae mihi talis videtur fuisse, ut, cum ostendere velles quales di essent, ostenderes nullos esse. a consuetudine oculorum animum abducere difficillimum dicebas, sed, cum deo nihil praestantius esset, non dubitabas quin mundus esset deus, quo nihil in rerum natura melius esset: modo possemus eum animantem cogitare vel potius ut cetera oculis sic animo hoc cernere [...] sin antem id dicis, nihil esse mundo sapientius, nullo modo prorsus adsentior, non quod difficile sit mentem ab oculis sevocare, sed quo magis sevoco eo minus id quod tu vis possum mente comprendere (ND 3.20-21).

Visto che in tutta la questione hai distinto quattro momenti e del primo si è già parlato, non ci resta che considerare il secondo. A me però sembra che esso abbia sortito un solo effetto, quello di far sì che proprio nel momento in cui ti sforzavi di chiarire la natura degli dèi ne dimostravi implicitamente l'inesistenza. Che sia molto difficile astrarre il pensiero dalle sensazioni visive sei stato tu a dirlo, ma non hai poi esitato a sostenere, partendo dal concetto della superiorità assoluta della divinità, che il mondo si identifica con essa divinità per il solo fatto che nulla vi è nella realtà di superiore al mondo. [...] Se però il senso delle tue parole è che nulla è più saggio del mondo, non posso assolutamente seguirti e, bada bene, non già 
perché sia difficile astrarre il pensiero dalle sensazioni visive, bensì perché quanto più cerco di astrarlo, tanto meno riesco ad afferrare il tuo pensiero. (trad. U. Pizzani) analisi, nega l'esistenza degli dèi, e fallisce nel compito di dimostrare la separazione fra gli occhi della mente e l'abitudine degli occhi, poiché, in ultima analisi, la divinità stoica non è nient'altro che il mondo-universo che percepiamo con gli occhi. È un dio visibile e materiale. D'altra parte, che cosa accade agli dèi quando si volgono verso il mondo visibile, o il mondo visibile si volge verso la divinità? Sono ancora dèi de facto? E ancora, se il mondo visibile non è la divinità, ma la sua immagine e somiglianza, questo significa avere un dio non visibile. Se così fosse, come sarebbe questa divinità, e dove si troverebbe?

Cotta presenta qui un problema epistemologico nella dottrina stoica, che già si era profilato nel Lucullus ${ }^{54}$. Per il protagonista di quel dialogo, l'essere umano è investito da ciò che vede, distinguendo due aspetti della conoscenza sensibile: quello passivo (phantasia-visum, derivato dalle percezioni dei sensi) e quello attivo, l'assentimento della percezione accolta come veritiera ${ }^{55}$. Lucullo dichiara che, nella mente umana, si formano visa di oggetti incorporei e altre realtà mentali. Sono «immagini non sensibili», che Cicerone non contraddice, nonostante il suo dissenso rispetto alla teologia stoica e le sue riserve sulla «accettazione delle percezioni autentiche» (synkatathesis) difesa dagli Stoici ${ }^{56}$.

Critica la nozione stoica degli dèi tradizionali, che fa di loro nient'altro che personificazioni di forze della natura benefiche nei confronti degli umani o di astrazioni morali. In questo modo, le statue e le altre figurazioni degli dèi sono teologicamente squalificate. Se le statue divine non sono che allegorie, giustificate solo in quanto permettono agli incolti e ai barbari di formarsi qualche conoscenza a proposito degli dèi, allora gli dèi romani non sono più dèi nel senso proprio del termine: il che non può essere accettabile per un pontefice e senatore come Cotta. Ma, d'altronde, la razionalità di Balbo non soddisfa i criteri accademici di verosimiglianza, per il suo apriorismo e il suo universalismo, e Cotta, richiamando polemicamente il vulgus, i Siri, gli Egizi e i Greci, chiede: «Questo è proprio degli incolti: e voi, filosofi, pensate qualcosa di meglio?» (haec igitur indocti; quid vos philosophi, qui meliora?) ${ }^{57}$.

Per Cotta, la teologia stoica è incongruente con la religione romana, e non rappresenta una spiegazione logicamente valida e persuasiva dei contenuti religiosi tradizionali. Soprattutto, la visione umana e le immagini divine sono valorizzate positivamente da Cotta nel processo di comprensione del divino: gli dèi sono parti integranti del mondo, e ciò che sappiamo di essi dipende dal nostro coinvolgimento con le immagini che li rappresentano. Se la conoscenza degli dèi dipende dalle «immagini non sensibili», queste dipendono dalla vita religiosa in questo mondo, che include le statue degli dèi, e l'abitudine degli occhi assolve un ruolo significativo nella costruzione mentale del divino. 


\section{Conclusione}

Per Cicerone, le immagini dipendono da chi le vede e dal contesto nel quale vengono viste. Le immagini divine sono, in ultima analisi, l'aspetto reale della presenza degli dèi nelle vite degli uomini. In generale, se Cicerone si appropria dell'esperienza dei pensatori che lo hanno preceduto, egli si misura in maniera ravvicinata con le teorie sulla dimensione fisica della conoscenza. Nei dialoghi filosofici, tradizione e ragione si rafforzano a vicenda. Le opere di Cicerone consolidano, modificano o respingono aspetti del pensiero greco, difendendo valori e pratiche tradizionali di fronte alle sfide intellettuali e politiche della sua epoca, o proponendo significative modifiche. Nei tre esempi presentati qui sinteticamente, la conoscenza e l'azione umana sono collegati alle statue degli dèi. La mente umana, come lo scultore, è limitata da quello che dispone nelle circostanze - materiali, quantità, durevolezza, colori - ma, se con questi elementi limitati lo scultore può creare effetti stupendi, anche la mente umana può aspirare ad attingere a una comprensione sicura del mondo e degli dèi. ${ }^{58}$ Cicerone difende l'unità della conoscenza, sia essa espressa nel linguaggio verbale o in quello delle arti figurative. L'oratoria e la filosofia sono modalità di creazione e di comunicazione che conducono l'essere umano alla conoscenza verosimile del mondo e degli dèi.

Nella Hermathena della villa di Tuscolo, la statua della dea è parte di una vera e propria estetica visiva e intellettuale della costruzione della Academia di Cicerone. Un'analogia proposta nel De Finibus fra la creazione di una statua da parte di Fidia e la costruzione estetico-intellettuale del sé è rivelatrice:

ut Phidias potest a primo instituere signum idque perficere, potest ab alio inchoatum accipere et absolvere, huic est sapientia similis; non enim ipsa genuit hominem, sed accepit a natura inchoatum. hanc ergo intuens debet institutum illud quasi signum absolvere (Fin. 4.34)

Ad esempio, Fidia può impostare una statua da principio e portarla alla fine, può riceverla già abbozzata da un altro e renderla perfetta; a lui è paragonabile la sapienza: non è lei che ha generato l'uomo, ma lo ha ricevuto già abbozzato dalla natura. Quindi, tenendo lo sguardo su questa, deve per così dire rendere perfetta quella statua già impostata. (trad. N. Marinone)

31 In ciò che riguarda la pulchra Libertas animata nella De Domo, la statua di Clodio agisce negativamente nel discorso - poiché la dea-statua invade con violenza la casa di Cicerone, che agisce maleficamente nella città non solo contro gli essere umani, ma anche contro le statue divine legittime. È visibile e agisce direttamente nel mondo fisico, sociale e religioso degli uomini. La statua è ovviamente un elemento dell'invettiva di Cicerone contro Clodio, ma la sua efficacia dipende da una credenza saldamente radicata nelle azioni delle statue degli dèi nel mondo.

32 Nel De natura deorum, il personaggio di Cotta sottolinea come i sensi ci pongano in contatto con le cose del mondo come esse ci appaiono: percepiamo visivamente immagini che non sono le cose in sé stesse, e la Nuova Accademia mette in dubbio l'affidabilità stessa dei sensi, ma la visione offre piste di indagine intorno alle cose del mondo. Se le condizioni di luminosità e di altro genere determinano la nostra percezione delle immagini, e senza che in esse vi sia alcuna stabilità, la visione permette di formare impressioni corrette sugli esseri e gli oggetti del mondo. Si possono inoltre fare 
affermazioni su questi esseri e questi oggetti, fondate su tali percezioni, ma in nessun caso si può avere piena certezza. Se la visione risulta poco chiara o approssimativa, questo dipende da molti fattori che incidono sull'interazione fra la cosa vista e la persona che la vede ${ }^{59}$. In ciò che riguarda le statue degli dèi, se esse non consentono di stabilire una base sicura per il iudicium veritatis ${ }^{60}, \mathrm{o}$, per così dire, un criterio di verità pura per le percezioni visive, permettono tuttavia che il giudizio umano si approssimi al verosimile nella comprensione della natura degli dèi ${ }^{11}$. In sintesi, Cicerone fonda fermamente la sua discussione sulla realtà empirica: sulla plasticità della rappresentazione degli dèi e sulla sua polisemia.

Vi sono profondamente integrate le impressioni visive, la percezione e la conoscenza, e tale unità non è molto distante dalla nostra comprensione del nostro 'modo di vedere' la realtà, come si dice in linguaggio moderno. Le statue degli dei sono componenti centrali nella costruzione del divino, attraverso la percezione sensoriale e l'evocazione verbale, e hanno la capacità di conferire presenza a cose che non esistono nel mondo sensibile, ma sono accessibili agli esseri umani grazie all'arte di dare forma materiale a un'idea. Il pensiero è anch'esso riflesso, costruito e dibattuto attraverso gli oggetti visibili, e il vocabolario filosofico di Cicerone copre un ampio spettro semantico fra l'atto di vedere, ciò che è visto, e la conoscenza che di questo rapporto si acquisisce. Cicerone riassume questi differenti aspetti in una formula: imago animi vultus, indices oculi ${ }^{62}$.

Le divinità sono viste, concepite e pensate in quanto statue, come ha dimostrato Peter Stewart e come anche Jaś Elsner ha sostenuto, da ultimo, nella sua discussione dei mutamenti che portarono al predominio dell'arte bidimensionale nel cristianesimo delle origini: le statue degli dèi erano troppo pericolose ${ }^{63}$. Nella tradizione religiosa occidentale, le statue sono ora apprezzate, ora vilipese, ma mai assenti. Michael Squire richiama un dipinto del 1580 ca., Il Trionfo del Cristianesimo, di Tommaso Laureti, nella Sala di Costantino in Vaticano ${ }^{64}:$ Gesù Cristo vi è presentato come la «incarnazione definitiva della divinità», e Squire si chiede se nella composizione il Cristo sia rappresentato o sia presente, mentre trionfa sulla statua ridotta in frantumi di un dio dall'aspetto apollineo. Si distingue dunque il 'trionfo cristiano', qui presentato come una lotta fra statue: l'immagine cristiana distrugge quella rivale. Le statue antropomorfe furono, e sono tuttora, uno strumento potentissimo per la costruzione del divino, che conferisce forza sia all'accesso religioso alla divinità che ai suoi usi politici ed estetici, e ai grandi conflitti che ne derivano ${ }^{65}$. La statua di un dio, peraltro, si presta a diversi usi e a funzioni complesse. Lo studio ravvicinato del lessico impiegato da Cicerone dimostra come essa possa acquisire un senso estetico, morale e intellettuale, espresso ed apprezzato dal punto di vista religioso. Le considerazioni di Cicerone sulle statue discusse in questo lavoro rivelano i nessi fra l'oggetto-statua e la divinità, e il suo impatto sul luogo in cui si trova l'immagine. La statua-divinità agisce sugli esseri umani, suscitando modalità di visione e di comprensione del divino ${ }^{66}$. 


\section{BIBLIOGRAFIA}

ADRYCH, DALGLISH 2020: P. Adrych, D. Dalglish, «Writing the Art, Archaeology and Religion of the Roman Mediterranean», in J. Elsner (ed.), Empires of Faith in Late Antiquity. Histories of Art and Religion from India to Ireland, Cambridge 2020, 51-79.

ALGRA 2003: K. Algra, «Stoic Theology», in B. Inwood (ed.), The Cambridge Companion to the Stoics, Cambridge 2003, 153-78.

ANDo 2008: C. Ando, The Matter of the Gods, Religion and the Roman Empire, Berkeley 2008.

ANDo 2010: C. Ando, «Praesentia numinis. Part 1: The Visibility of Roman Gods», Asdiwal 5 (2010), 45-73.

ARENA 2020: V. Arena, «Cicero, the Augures, and the Commonwealth», in C. Beltrão, F. Santangelo (eds.), Cicero and Roman Religion. Eight Studies, Stuttgart 2020, 23-43.

ARENA 2012: V. Arena, Libertas and the Practice of Politics in the Late Roman Republic, Cambridge 2012.

BARASCH 2001: M. Barasch, «The Idol in the Icon: Some Ambiguities», in J. Assmann, A. I. Baumgarten (eds.) Representation in Religion, Studies in Honor of Moshe Barash, Leiden 2001, 1-25. BELAYCHE, ESTIENNE 2020: N. Belayche, S. Estienne, «L'autel et la toge: Entrée en matière», in N. Belayche, S. Estienne (dir.), Religion et pouvoir dans le monde romain. L'autel et la toge, Rennes 2020, 11-34.

BELAYCHE, BRULÉ 2010: N. Belayche, P. Brulé, «Introduction: Nomination et représentation du divin», ARG 12 (2010), 3-5.

Belayche, PirenNe-Delforge 2015: N. Belayche, V. Pirenne-Delforge, «Fabriquer du divin: en guise de prélude», in N. Belayche, V. Pirenne-Delforge (eds.), Fabriquer du divin, Constructions et ajustements de la représentation des dieux dans l'Antiquité, Liège 2015, 7-20.

BELTRÃo 2002: C. Beltrão, «Uma leitura helenística do pensamento aristotélico: oikeiosis e justiça em Antíoco de Ascalão», Phoînix 8 (2002), 95-114.

BELTRÃo 2018: C. Beltrão, «Imágenes de los dioses en Cicerón: Límites y contradicciones de la teología epicúrea sobre la imagen divina en De natura deorum I», Auster (2018), 9-20.

Beltrão 2020: C. Beltrão, «The God and the Consul in Cicero's Third Catilinarian», in C. Beltrão, F. Santangelo (eds.), Cicero and Roman Religion. Eight Studies, Stuttgart 2020, 45-58.

Begemann 2015: E. Begemann, « 'Ista tua Pulchra Libertas': The Construction of a Private Cult of Liberty on the Palatine», in C. Ando, J. Rüpke (eds.), Public and Private in Ancient Mediterranean Law and Religion, Berlin 2015, 75-98.

BERTHELET 2016: Y. Berthelet, «La consecratio du terrain de la domus palatine de Cicéron», MEFRA 128.2 (2016), 457-468.

BERTHELET 2020: Y. Berthelet, «De la difference entre l'auctoritas des prêtres et celles de magistrats sous la République romaine», in J.-M. David, F. Hurlet (eds.), L'auctoritas à Rome. Une notion constitutive de la culture politique, Bordeaux 2020, 121-143.

BowEs 2015: K. Bowes, «At Home», in R. Raja, J. Rüpke (eds.), A Companion to the Archaeology of Religion in the Ancient World, Maiden, MA 2015, 209-215. 
BRITTAIN 2001: C. Brittain, Philo of Larissa. The Last of the Academic Sceptics, Oxford 2001.

BRITTAIN 2005: C. Brittain, «Common Sense: Concepts, Definition and Meaning In and Out of the Stoa», in B. Inwood, D. Frede (eds.) Language and Learning: Philosophy of Language in the Hellenistic Age, Cambridge 2005, 164-209.

CAIRo 2018: M. E. Cairo, «El debate en torno a Libertas a fines da República: una lectura de De domo sua de Cicerón», Phoînix 24.2 (2018), 75-98.

CAIRO 2020a: M. E. Cairo, «Aproximaciones a la identidad romana en los discursos post reditum de Cicerón», Politica Antica 10 (2020), 65-80.

CAIRO 2020b: M. E. Cairo, «Libertatis simulacrum (dom. 110), simulacrum non libertatis (dom. 131): Reflections on the Nature of the Images of the Gods in Cicero», in C. Beltrão, F. Santangelo (eds.), Estátuas na religião romana, Coimbra 2020, 65-80.

Chaniotis 2017: A. Chaniotis, «The Life of Statues: Emotion and Agency», in D. Cairns, D. Nelis (eds.), Emotions in the Classical World. Methods, Approaches, and Directions, Stuttgart 2017, 141-158.

ChANTRAINE 1968: P. Chantraine, Dictionnaire étymologique de la langue grecque: histoire des mots, Paris 1968.

CLARK 2007: A. J. Clark, Divine Qualities. Cult and Community in Republican Rome, Oxford 2007.

DRoogan 2013: J. Droogan, Religion, Material Culture, and Archaeology, New York 2013.

ELSNER 1996: J. Elsner, «Image and Ritual: Reflections on the Religious Appreciation of Classical Art», Classical Quarterly 42.2 (1996), 515-531.

ELSNER 2007: J. Elsner, Roman Eyes: Visuality and Subjectivity in Art and Text, Princeton 2007.

ELSNER 2020a: J. Elsner, «Introduction», in J. Elsner (ed.), Empires of Faith in Late Antiquity. Histories of Art and Religion from India to Ireland, Cambridge 2020, 1-23.

ELSNER 2020b: J. Elsner, «A Estatueta Animada na Arqueologia Mediterrânea: o fim de uma história», in C. Beltrão, F. Santangelo (eds.), Estátuas na religião romana, Coimbra 2020, 159-182.

ELSNER, SQUIRE 2016: J. Elsner, M. Squire, «Sight and Memory. The Visual Art of Roman Mnemonics», in M. Squire (ed.), Sight and the Ancient Senses, London-New York 2016, 180-204.

EstienNe, Huet, LISSARRAgUe, Prost 2014: S. Estienne, V. Huet, F. Lissarrague, F. Prost (dir.), Figures de dieux: construire le divin en images, Rennes 2014.

ESTIENNE 2000: S. Estienne, Les Dieux dans la ville: recherches sur les statues de dieux dans l'espace et les rites publiques de Rome, d'Auguste à Severe Alexandre (I ${ }^{e r}-I I I^{e}$ s.a.J.-C). Diss. Paris 2000.

ESTIENNE 2010: S. Estienne, «Simulacra deorum versus ornamenta aedium: the status of divine images in the temples of Rome», in J. Mylonopoulos (ed.), Divine Images and Human Imaginations in Ancient Greece and Rome, Leiden-Boston 2010, 257-271.

ESTIENNE 2014: S. Estienne, «Aurea pompa venit. Présences divines dans les processions romaines», in S. Estienne, V. Huet, F. Lissarrague, F. Prost (eds.), Figures de dieux: construire le divin en images, Rennes 2014, 337-349.

FARAONE 1992: C. Faraone, Talismans and Trojan Horses: Guardian Statues in Ancient Greek Myth and Ritual, Oxford 1992.

FARAONE 2017: C. Faraone, «A Wax Effigy Pierced by Three Bones: The Pharaonic Origins of a Late Antique Cursing Ritual?», Symbolae Osloenses 91 (2017), 126-33. 
FRANKFURTER 2018: D. Frankfurter, Christianizing Egypt: Syncretism and Local Worlds in Late Antiquity, Princeton 2018.

GILDENHARD 2011: I. Gildenhard, Creative Eloquence. The Construction of Reality in Cicero's Speeches, Oxford 2011.

GLUCKER 1996: J. Glucker, «Consuetudo oculorum», in R. Katzoff et al. (eds.), Classical Studies in Honor of David Sohlberg, Ramat Gan 1996, 105-23.

GLUCKER 2020: J. Glucker, Classics and Classicists: Selected Essays 1964-2000, ed. A. Edelheit, Newcastle upon Tyne 2020 .

GORDON 1979: R. Gordon, «The Real and the Imaginary: Production and Religion in the GraecoRoman World», Art History 2 (1979), 5-34.

HÖLKESKAMP 2004: K.-J. Hölkeskamp, «Under Roman Roofs: Family, House, and Household», in H. I. Flower (ed.), The Cambridge Companion to the Roman Republic, Cambridge 2004, 113-138.

JENSEN 2013: R. M. Jensen, «Visuality», in B. S. Spaeth (ed.), The Cambridge Companion to Ancient Mediterranean Religions, Cambridge 2013, 309-343.

JONES 2016: L. Jones, «Memory, Nostalgia, and the Roman Home», in M. G. Morcillo, J. H. Richardson, F. Santangelo (eds.), Ruin or Renewal? Places and the Transformation of Memory in the City of Rome, Roma 2016, 183-211.

KenTY 2018: J. Kenty, «The Political Context of Cicero's Oration De domo sua», Ciceroniana online 2.2 (2018), 245-264.

KLEVE 1978: K. Kleve, «On the Beauty of God. A Discussion between Epicureans, Stoics and Sceptics», Symbolae Osloenses (1978), 69-83.

KÖSTER 2017: I. Köster, «Sacred Objects, Material Value, and Invective in Cicero's Verrines II 4», in S. Blakely (ed.), Gods, Objects, and Ritual Practice, Atlanta 2017, 151-167.

KOUSSER 2017: R. Kousser, The Afterlives of Greek Sculpture: Interaction, Transformation and Destruction, Cambridge 2017.

LEEN 1991: A. Leen, «Cicero and the Rhetoric of Art», The American Journal of Philology 112.2 (1991), 229-245.

LENNON 2010: J. Lennon, «Pollution and Ritual Impurity in Cicero's De domo sua», Classical Quarterly 60.2 (2010), 427-445.

LISDORF 2005: A. Lisdorf, «The Conflict over Cicero's House: An Analysis of the Ritual Element in De Domo Sua», Numen 52.4 (2005), 445-464.

LoNG 1996: A. A. Long, Stoic Studies, Cambridge 1996.

MACRAE 2016: D. MacRae, Legible Religion. Books, Gods, and Rituals in Roman Culture, Cambridge-Mass. 2016.

Malamoud, Vernant 1986: Ch. Malamoud, J.-P. Vernant (dir.), Corps des dieux, Paris 1986.

MAAR 2004: C. Maar, Iconic turn: die neue Macht der Bilder, Köln 2004.

MiLEs 2008: M. M. Miles, Art as Plunder. The Ancient Origins of Debate about Cultural Property, Cambridge 2008.

MIRZOEFF 2002: N. Mirzoeff (ed.), The Visual Culture Reader, London 2002. 
MoтTA 2018: A. Motta, «The Philosophy of Artistic Creation: Phidias, the Ideas and Cicero», Apeiron 51.3 (2018), 1-20.

MYLONOPOULOS 2010: J. Mylonopoulos, «Introduction - Divine Images versus Cult Images. An Endless Story about Theories, Methods, and Terminologies», in J. Mylonopoulos (ed.), Divine Images and Human Imaginations in Ancient Greece and Rome, Leiden-Boston 2010, 1-19.

Nightingale 2016: A. Nightingale, «Sight and the Philosophy of Vision in Classical Greece. Democritus, Plato and Aristotle», in M. Squire (ed.), Sight and the Ancient Senses, London-New York 2016, 54-67.

NORTH 2014: J. A. North, «The Limits of the 'Religious' in the Late Roman Republic», History of Religions 53 (2014), 225-245.

PinA Polo 2002: F. Pina Polo, «Cicerón, elegido de los dioses: la reprobación religiosa del adversario político como recurso retórico», in F. Pina Polo, J. Remesal Rodríguez (eds.), Religión y propaganda política en el mundo romano, Barcelona 2002, 57-69.

RigGSBY 2002: A. M. Riggsby, «The Post Reditum Speeches», in J. M. May (ed.), Brill's Companion to Cicero. Oratory and Rhetoric, Leiden 2002, 159-196.

RÜPKE 2010: J. Rüpke, «Representation or Presence? Picturing the Divine in Ancient Rome», Archiv für Religionsgeschichte 12 (2010), 181-196.

RÜPKE 2012: J. Rüpke, Religion in Republican Rome: Rationalization and Ritual Change, Philadelphia 2012.

RÜPKE 2015: J. Rüpke, «The Role of Priests in Constructing the Divine in Ancient Rome», in N. Belayche, V. Pirenne-Delforge (eds.), Fabriquer du divin, Constructions et ajustements de la représentation des dieux dans l'Antiquité, Liège 2015, 79-92.

RÜPKE 2019: J. Rüpke, «Roman Gods and Private Property. The Invention of State Religion in Cicero's On His House», Religion in the Roman Empire 5 (2019), 292-315.

RÜPKE 2020: J. Rüpke, «Prêtres romains: sources et limites de l'autorité religieuse», in N. Belayche, S. Estienne (dir.), Religion et pouvoir dans le monde romain. L'autel et la toge, Rennes 2020, 37-53.

SALLER 2013: R. P. Saller, Patriarchy, Property, and Death in the Roman Family, Cambridge 1994.

SANTANGElo 2013a: F. Santangelo, Divination, Prediction and the End of Roman Republic, Cambridge 2013.

SANTANGELO 2013b: F. Santangelo, «Priestly auctoritas in the Roman Republic», Classical Quarterly 63 (2013), 743-763.

SCHEIDEGGER-Lämmle 2017: C. Scheidegger-Lämmle, «On Cicero's De domo sua: A Survey of Recent Work», Ciceroniana online 1 (2017), 147-156.

SCHOFIELD 1980: M. Schofield, «Preconception, Argument and God», in M. Schofield, M. Burnyeat, J. Barnes (eds.) Doubt and Dogmatism: Studies in Hellenistic Epistemology, Oxford 1980, 283-308. SEDLEY 2012: D. Sedley (ed.), The Philosophy of Antiochus, Cambridge 2012.

SEDLEY 2013: D. Sedley, «Cicero and the Timaeus», in M. Schofield (ed.), Aristotle, Plato and Pythagoreanism in the First Century BC: New Directions for Philosophy, Cambridge 2013, 187-205. SQUIRE 2009: M. Squire, Image and Text in Graeco-Roman Antiquity, Cambridge 2009.

SQUIRE 2011: M. Squire, The Art of the Body: Antiquity and its Legacy, London-New York 2011. 
STEINER 2001: D. Steiner, Images in Mind: Statues in Archaic and Classical Greek Literature and Thought, Princeton 2001.

STEWART 2003: P. Stewart, Statues in Roman Society: Representation and Response, Oxford 2003.

STRон 2004: W. Stroh, «De Domo Sua: Legal Problem and Structure», in J. Powell, J. Paterson (eds.), Cicero the Advocate, Oxford 2004, 313-370.

TANNER 2006: J. Tanner, The Invention of Art History in Ancient Greece. Religion, Society and Artistic Rationalisation, Cambridge 2006.

TARRANT 1985: H. Tarrant, Scepticism or Platonism? The Philosophy of the Fourth Academy, Cambridge, 1985.

TARRANT 2007: H. Tarrant, «Antiochus: A New Beginning?», in R. W. Sharpies-R. Sorabji (eds.), Greek and Roman Philosophy. 100 BC - 200 AD. V. 2. London 2007, 317-332.

TREGGIARI 1999: S. Treggiari, «The Upper-Class House as Symbol and Focus of Emotion in Cicero», JRA 12 (1999), 33-56.

VAN DER Plas 1987: D. van der Plas (ed.), Effigies Dei. Essays on the History of Religions, Leiden 1987, 156-170.

VAN HAEPEREN, BeRTHelet 2021: F. Van Haeperen, Y. Berthelet (dir.) Dieux de Rome et du monde romain en réseaux, Bordeaux 2021.

VAN NUfFELEN 2011: P. Van Nuffelen, Rethinking the Gods. Philosophical Readings of Religion in the PostHellenistic Period, Cambridge 2011.

VAN OUWERKERK 1987: C. A. J. van Ouwerkerk, «Effigies Dei and the Religious Imagination», in D. van der Plas (ed.), Effigies Dei. Essays on the History of Religions, Leiden 1987, 156-170.

WALLACE-HADRILL 1988: A. Wallace-Hadrill, «The Social Structure of the Roman House», PBSR 56 (1988), 43-97.

WILBURN 2012: A. Wilburn, Materia Magica: The Archaeology of Magic in Roman Egypt, Cyprus and Spain, Ann Arbor 2012.

WYNNE 2019: J. P. F. Wynne, Cicero on the Philosophy of Religion. On the nature of the Gods and On Divination, Cambridge 2019.

ZACCARIA RUGGIU 1995: A. Zaccaria Ruggiu, Spazio privato e spazio pubblico nella città romana, Roma 1995.

\section{NOTE}

1. Su questo punto vd. Belayche, BRUlé 2010; i contributi riuniti in estienNe, hUET, LISSARRAGUe, PRost 2014; e, soprattutto, BeLAyche, PirenNe-Delforge 2015, 7-20. Vd. inoltre il recente volume collettivo a cura di VAN HAEPEREN, BERTHELET 2021, che comprende studi su diversi tentativi di concettualizzare e rappresentare il divino nella riflessione e nella prassi religiosa del Mediterraneo in età romana.

2. Le innovazioni portate da Cicerone e da altri autori romani al campo che comunemente si definisce «religione romana» sono state l'oggetto di un intenso dibattito. Vd. ad es. ANDO 2008, GILDENHARD 2011, RÜPKE, 2012, SANTANGELO 2013a, NORTH 2014 e MACRAE 2016.

3. TANNER 2006, 31-96 discute i rapporti fra il linguaggio dell'arte, lo sviluppo del presentational style delle statue degli dèi, il vocabolario religioso e le risposte religiose ed estetiche alle immagini in 
ambito sacro. Vd. STEINER 2001, un punto di riferimento fondamentale sulle strategie attraverso le quali gli autori greci dei periodi arcaico e classico concettualizzarono le sculture. Vd. anche SQUIRE 2009, 111-120. Riguardo al caso romano, vd. ELSNER 2007, spec. la Parte I, sui parametri artistici e letterari del processo di ritual viewing, dal periodo classico alla tarda antichità, in vari contesti.

4. SQUIRE 2011 è un'eccellente introduzione alle questioni poste dall'immagine antropomorfica. Due contributi fondamentali sul tema dell'immagine divine sono GORDON 1979 e ELSNER 1996, che sostiene l'inscindibilità dell'apprezzamento estetico da quello religioso nell'antichità classica, oltre ai fondamentali studi riuniti in MALAMOUD, VERNANT 1986.

5. Vd. spec. ELSNER 2020a e ADRYCH, DALGLISH 2020.

6. Vd. VAN DER PlAS 1987, in part. VAN OUWERKERK 1987. In anni più recenti, vd. STEWART 2003, 184-222, RÜPKE 2010, e CHANIOTIS 2017, spec. 149-153.

7. RÜPKE 2010.

8. Vd. spec. ANDO 2008, 21-42.

9. Il dinamismo delle immagini e il tema della rappresentazione sono aspetti capitali dello studio della cultura visuale: intendono l'immagine come un'evidenza storica degli atti visivi, e la visione stessa come una forma di pensiero e di azione, fondata in pratiche sociali fermamente localizzate che costruiscono la realtà. Sugli studi della cultura visiva vd ad. es. i lavori riuniti in MiRzoEfF 2002 e MAAR 2004. Per una visione generale del tema in rapporto alla storia delle religioni nel Mediterraneo antico vd. JENSEN 2013. Vd. anche BARASCH 2001 sulle religioni abramitiche.

10. Vd. ANDO 2010 e R ÜPKE 2010, che hanno dimostrato come le teorie moderne della rappresentazione o dell'iconicità non siano sempre adatte allo studio delle statue degli dèi nel mondo romano. $\mathrm{Vd}$. anche RÜPKE 2015.

11. GORDON 1979 E STEINER 2004, 79.

12. Vd. STEWART 2003, spec. 203-231. Cf. ad es. Varrone, RD fr. 228 Cardauns, sugli dèi, i loro templi e i segni ai quali ricorrono, e Cicerone, ND 1.81, che sottolinea il ruolo dell'oggetto visibile nel processo attraverso cui gli dèi vengono conosciuti e rappresentati.

13. Vd. la discussione in Mylonopoulos, 2010. Sul contesto romano e i problemi concettuali dell'idea (moderna) di statua cultuale, sugli ornamenta e sui loro rapporti con le statue degli dèi, vd. ESTIENNE 2010.

14. Sull'aspetto animato e l'azione delle statue nel mondo greco-romano, vd. FARAONE 1992, Wilburn 2012, 56-83, KoUSSER 2017, F ARAONE, 2017, FRANKFURTER 2018, 24-38, 56-66, 127-130, e ELSNER 2020b.

15. Vd. spec. Att. 1.1.5, 1.4.3, 1.6.2, 1.8.2, 1.9.1, 1.9.2, 1.10.3, 2.8.2, 2.9.4, 4.2.5-7, 12.4.1, 13.9, e Fam. 7.23.2. La maggior parte di questi riferimenti indica come Attico, in particolare, abbia acquistato molte opere per, o per conto di, Cicerone, e abbia anche organizzato la collezione d'arte di Pompeo nel 55 a.C.: Att. 4.9.9.

16. Cf. Tusc. 2.9; Div. 1.8 .

17. Cf. ad es. Leg. 2.3-4, sulla casa avita di Arpino, e Dom. 108-109, sulla domus del cittadino romano. Per alcune importanti discussioni sul tema vd. WALLACE-HADRILL 1988, SALLER 1994, 80-95, ZACCARIA RUGgiU 1995, spec. 311-347, TREgGIARI 1999, HöLKESKAMP 2004 e JONES 2016. LEEN 1991 è particolarmente rilevante per una comprensione più approfondita dei rapporti fra ciò che viene definito 'uso retorico' dell'arte e la strategia di autorappresentazione di Cicerone e dei suoi contemporanei, fondati sulla persona pubblica e sull'ethos del suo proprietario. Vd. anche MILES $2008,152-217$, che si concentra in particolare sul mercato dell'arte.

18. Vd. StewARt 2003, 184-222 per le definizioni dei termini relativi alle statue divine. Per uno studio più dettagliato del vocabolario delle immagini divine, vd. EsTIENNE 2000.

19. La discussione della cosiddetta interpretatio Romana non rientra nel tema di questo articolo, ma l'atto di conferire nuovi significati alla divinità rinominata ne è un aspetto significativo. 


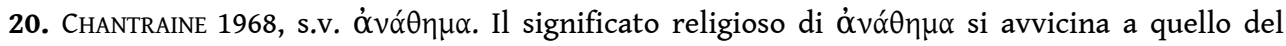
termine latino sacer, nel senso della remissione di un oggetto o di un individuo dedicato agli dèi, e dunque qualcuno o qualcosa che viene escluso dal consorzio degli esseri umani e dalla vita quotidiana.

21. Vd. Mylonopoulos 2010, ESTIENNe 2010, Droogan 2013, 149-173, e Bowes 2015.

22. Vd. ad es. KöSTER 2017 sulla ritualizzazione delle opere d'arte e delle statue operata da Cicerone, che le trasforma in oggetti divini attraverso la strategia retorica perseguita nel De signis (Verr. 2.4).

23. Tusc. 2.3. Vd. anche Fam. 9.6.4 sulla villa di Tuscolo e il suo significato intellettuale.

24. Sul conflitto politico della De Domo vd. ARENA 2012, 200-220. Altre importanti discussioni della dimensione religiosa del conflitto fra Cicerone e Clodio: LISDORF 2005, GILDENHARD 2011, 300-326, BEGEMANN 2015, CAIRO 2018, e RÜPKE 2019, dove si analizzano le argomentazioni alle quali Cicerone fece ricorso nel tentativo di recuperare l'uso della sua casa, a dispetto del fatto che il suo pubblico condividesse l'idea per cui un trasferimento correttamente portato a termine non avrebbe potuto essere sottratto agli dèi. Vd. anche SCHEIDEGGER-LÄMMLE 2017. Questi studiosi dimostrarono che, se per molto tempo la De Domo fu considerata un testo secondario nel corpus ciceroniano e, al contempo, di qualità inferiore alla maggior parte delle opere di questo autore, è stata recentemente riconsiderata, facendo giustizia a ciò che Cicerone disse ad Attico in Att. 4.2.2 sull'alta qualità del discorso.

25. Cf. Har. resp. 11. Vd. Plut. Cic. 33.4, e STROH 2004, 323, che sottolinea la fragilità del caso dal punto di vista giuridico e religioso, in una situazione politica molto difficile per Cicerone. Su questo punto vd. anche KENTY 2018.

26. Dom. 51, sulla mozione di Bibulo e le possibili accuse di impedimenti religiosi riguardanti il terreno della casa del Palatino.

27. Sullo stretto rapporto fra religione e politica nella Repubblica romana vd. ad es. SANTANGELO 2013b, BERTHELET 2020, BELAYCHE, ESTIENNE 2020, e RÜPKE 2020.

28. Le case di Cicerone attaccate da Clodio erano la domus sul Palatino e le villae di Tuscolo e di Formia. La casa avita di Arpino e la domus urbana alle Carinae, presso il tempio di Tellus, dove risiedeva Quinto Cicerone, non subirono danni (Dom. 62, incluse le insulae sull'Argileto e sull'Aventino).

29. Vd. spec. BERTHELET 2016.

30. La De domo, come è noto, fa parte della serie dei discorsi detti post reditum, pronunciati fra il settembre del 57 e il maggio del 56 a.C., subito dopo il ritorno a Roma dall'esilio. Si trattava di un passaggio cruciale per Cicerone, che cercava di riguadagnare non soltanto le sue proprietà, ma anche la sua posizione nel quadro politico romano. Sulle caratteristiche che accomunano questi discorsi vd. ad es. RIGgSBY 2002, PINA POLO 2002 e, da ultimo, CAIRO 2020a.

31. Vd. spec. LENNON 2010 e GILDENHARD 2011, 315-326.

32. Come sostiene GILDENHARD 2011, 312: «(T)he opening paragraphs thus prepare the ground for his emphasis on ius (broadly conceived as justice) and, by rejecting the orthodox power of religio on which Clodius insisted, implicitly raise the question as to what, precisely, divina religio is».

33. Dom. 51.

34. Vd. Cic. Verr. 2.4.64, che pone una statua al centro del processo di consacrazione di un santuario.

35. Vd. Clark 2007, 18, 22-24, 205-254 e ARENa 2012, 34-43.

36. CAIRO $2020 \mathrm{~b}$.

37. Dom. 110.

38. Dom. 111-113. Vd. CAIRO 2018.

39. Dom. 111. 
40. Ad es., in Dig. 1.8.6 (Marciano), si legge: sacrae autem res sunt hae, quae publice consecratae sunt, non private: si quis ergo privatim sibi constituerit sacrum «sacrum constituerit», sacrum non est, sed profanum. Si tratta di una testimonianza più tarda rispetto ai testi di cui ci si occupa in questo studio, ma comunque significativa, perché fa riferimento ai problemi posti dalla consecratio di Clodio. Vd., ad es., BeRTHELET 2016.

41. Dom. 138 .

42. Leg. 2.26. Gli anni Cinquanta e Quaranta furono segnati da profondi mutamenti politici e religiosi, nel quadro di un duro confronto di parte e un'aspra crisi istituzionale. La religione appare nel secondo libro del De Legibus come una risposta a un periodo di grandi trasformazioni a Roma e nell'Impero. Quando il personaggio di Marco sostiene che la «confusione« religione vada evitata, va tenuto presente che gli «dei particolari» (separatim nemo habessit deos: Leg. 2.19) a cui fa riferimento sono dèi nuovi, oltre che stranieri. Queste divinità sono opposte agli dèi tradizionali, ai quali sono riservati i culti pubblici, e agli dèi legati ai sacra della famiglia. Sulle innovazioni religiose di Cicerone nel De Legibus vd. spec. ARENA 2020. La Pulchra Libertas di Clodio emerge come una dea particolare, che genera confusione e turba sia Cicerone che la città nel suo complesso.

43. Spec. Cat. 3.20-22. Vd. GILDENHARD 2011, 272-292, BELTRÃo 2020. Vd. anche ELSNER 2020b, spec. 167-174, sul rapporto che viene stabilito nei testi letterari fra l'animazione delle statue di culto, l'emozione e l'azione: uno scenario possibile solo se si accetta il presupposto che le statue degli dèi siano effettivamente in grado di compiere tali azioni.

44. Div. 1.19, 2.45-47.

45. La portata della riflessione teologica di Cicerone è stata recentemente ribadita da WYNNE 2019, che si concentra sull'analisi dei dialoghi De Natura Deorum e De Divinatione come elementichiave di un progetto volto a costituire una filosofia della religione.

46. $N D$ 1.81. Vd. anche ND 1.90, dove Cotta critica la tesi epicurea per cui gli dèi sarebbero somiglianti agli esseri umani?: «poiché gli dèi sono sempre esistiti, non sono mai nati; se davvero sono eterni, sempre esisteranno. Al contrario, gli uomini nasceranno, e dunque la forma umana è esistita prima degli uomini e gli dèi immortali avevano questa forma: la forma umana perciò non è la loro, ma occorre dire che la nostra forma è quella divina» (di enim semper fuerunt, nati numquam sunt, si quidem aeterni sunt futuri; at homines nati, ante igitur humana forma quam homines, eaque erant forma dii immortales, non ergo illorum humana forma sed nostra divina dicenda est). Per un'analisi della controversia fra Cotta e Velleio sulle immagini antropomorfiche degli dèi vd. BELTRÃo 2018.

47. ND 2.118 .

48. ND 2.58. Vd. l'analisi dei discorsi di Velleio e di Balbo in WYNNE 2019, risp. 83-110, e 138-164. Sulla questione teologica della bellezza divina vd. spec. KLEVE 1978.

49. Vd. spec. ND 2.156.

50. ND 2.5. Vd. SCHOFIELD 1980 e BRITTAIN 2005.

51. Cfr. Lucullus 21, 27, 78, 98, 108. Sul pensiero di Antioco di Ascalona vd. BeltrÃo 2002, TARRANT 2007, e, soprattutto, gli studi raccolti in SEDLEY 2012. Sulla questione del debito dello scetticismo accademico verso la tradizione platonica vd. TARRANT 1985. Sul tema della consuetudo oculorum vd. GLUCKER 1996 (= 2020, 106-123).

52. ND 2.66-72.

53. La trattazione della teologia stoica è molto più ampia nel De natura deorum di quanto lo sia quella della teologia epicurea. La lunga discussione di Balbo nel secondo libro e il celebre «verdetto di Cicerone» nel finale del dialogo sembrano sostenere l'approvazione di una teologia stoica da parte di Cicerone. Peraltro, secondo SEDLEY 2013, 202, questo verdetto sarebbe ambiguo, poiché non sostiene che il discorso di Balbo è veritiero, ma che tende (propensior) ad veritatis similitudinem. Questa interpretazione appare molto produttiva anche alla luce dell'uso 
ciceroniano di similitudo, e porta dunque a concludere che il discorso di Balbo sia considerato lontano dalla veritas.

54. Ad es. Luc. 144-145.

55. Sulla dottrina stoica vd. ad es. LONG 1996; ALGRA 2013, e VAN NUffelen 2011. Sulla parziale accettazione da parte di Filone di Larissa delle definizioni stoiche della phantasia e della katalepsìa, la cui posizione viene difesa nel Lucullus dal personaggio di Cicerone: vd. BRITTAIN 2001, 220-225. Vd. anche Sext. Emp. Math. 8.397.

56. Luc. 21, 27. Secondo MotTA 2018, la nozione di «immagini non sensibili», o immagini mentali, può avere condotto Cicerone a formulare il concetto di cogitatio mentis per spiegare il processo creativo dell'arte. Vd. anche Luc. 78, 98, 108.

57. ND 2.59.

58. SQUIRE, ELSNER, 2016.

59. Per una discussione d'assieme sulla filosofia della visione nel mondo antico, vd. NightingaLE 2016.

60. Ac. 1.30 .

61. Luc. 111.

62. De Or. 3.221.4.

63. STEWART 2003, spec. 203-231 ed ELSNER 2020b.

64. SQUIRE 2011, 154-156.

65. Vd. spec. gli articoli compresi nella prima parte di EstienNe, HUEt, LisSARRAGUe, Prost 2014, e ESTIENNE 2014 e Belayche, PirenNe-Delforge 2015, 7-20.

66. Ringrazio Federico Santangelo per la lettura di una precedente versione di questo testo e per la sua traduzione italiana.

\section{RIASSUNTI}

Nell'opera di Cicerone si possono riconoscere tre modalità di approccio alle statue antropomorfe degli dèi. La prima intende la statua come un'oggettivazione della divinità. La seconda associa strettamente la statua all'entità divina, e contempla la nascita di un'entità animata. La terza modalità si concentra sulla questione teologica di come pensare gli dèi, nella loro immortalità, a partire da un oggetto materiale. Questo contributo si misura con ciascuno di questi modi di concepire e di riferirsi alle statue divine attraverso tre casi di studio: una statua nella villa di Cicerone a Tuscolo, una statua intorno alla quale si imperniò una disputa sul controllo dello spazio pubblico, e un breve passo tratto da una controversia filosofica sulla natura degli dèi. In tutti e tre i casi, l'immagine divina ha un valore estetico, morale e intellettuale significativo dal punto di vista religioso, che rivela i nessi fra statua e divinità, e i suoi effetti sul luogo in cui essa si trova e sugli esseri umani, creando modi di vedere e di comprendere il divino.

In Cicero's work three interpretive modes concerning divine figures or different approaches to anthropomorphic statues may be identified. The first one views the statue as the objectification of the deity. The second one envisages a blending of the statue and the deity into an animated being. The third mode focuses on the theological question of how to think of the immortal gods through a material object. This paper engages with these ways of conceiving and referring to divine statues by considering three case-studies: a statue in Cicero's Tusculan villa, a pivotal 
statue in a public speech, and a brief passage of a theological controversy on divine forms. In Cicero's discourse, the divine image has an aesthetic, moral, and intellectual value, which has deep religious significance, revealing the links between statue and deity, its effect on the place in which it is located, and its impact upon human beings enabling new ways of seeing and understanding the divine.

INDICE

Keywords : religious images, anthropomorphic statues, animated statues, divine knowledge, religious discourse

Parole chiave : immagini religiose, statue antropomorfe, statue animate, conoscenza divina, discorso religioso

\section{AUTORE}

\section{CLAUDIA BELTRÃO}

Universidade Federal do Estado do Rio de Janeiro,

Avenida Pasteur,458,

sala 216, Urca,

Rio de Janeiro 22290-240, Brasil,

claudia.rosa(at)unirio.br 